\title{
Management of Non-Healing Ulcers by Autologous Platelet Rich Fibrin (PRF)
}

\author{
Kartavya Chaudhari ${ }^{1,2,3}$, Parvez Ahmad Ganie ${ }^{1,2,3,4}$, Nilesh Kumar \\ Agrawal $^{5}$, Rashmi Jain ${ }^{6}$, Madhan Jeyaraman ${ }^{1,2,3,4 *}$ and Manish \\ Khanna ${ }^{1,2,3,7}$ \\ ${ }^{1}$ Fellow in Orthopaedic Rheumatology, Dr. Ram Manohar Lohiya National Law \\ University, Lucknow, Uttar Pradesh, India \\ ${ }^{2}$ Indian Stem Cell Study Group (ISCSG) Association, Lucknow, Uttar Pradesh, India \\ ${ }^{3}$ Indian Orthopaedic Rheumatology Association (IORA), Lucknow, Uttar Pradesh, \\ India \\ ${ }^{4}$ Department of Orthopaedics, School of Medical Sciences and Research, Sharda \\ University, Greater Noida, Uttar Pradesh, India \\ ${ }^{5}$ Department of Orthopaedics, JJM Medical College, Davangere, Karnataka, India \\ ${ }^{6}$ Resident, School of Medical Sciences and Research, Sharda University, Greater \\ Noida, Uttar Pradesh, India \\ ${ }^{7}$ Department of Orthopaedics, Prasad Institute of Medical Sciences, Lucknow, Uttar \\ Pradesh, India \\ *Corresponding Author: Madhan Jeyaraman, Department of Orthopaedics, School \\ of Medical Sciences and Research, Sharda University, Greater Noida, Uttar Pradesh, \\ India.
}

\author{
Received: July 23, 2021 \\ Published: August 04, 2021 \\ (C) All rights are reserved by Madhan \\ Jeyaraman., et al.
}

\begin{abstract}
Introduction: Chronic non-healing ulcer is defined as a persistent defect in the skin for the period of more than six weeks and does not exhibit any tendency to heal following three or more months. Non-healing ulcers are enfeebling and leads to dwindling of quality life of a patient. Venous diseases, arterial diseases, and neuropathy are some of the common causes of these non-healing ulcers. The underlying pathology for chronic ulceration is multifactorial which calls for a patient's systematic evaluation for the purpose of ascertaining etiology and optimization of the treatment. The therapeutic use of autologous platelet rich fibrin (PRF) represents a newer regenerative avenue to stimulate and accelerate complex wound healing. In this article, we aimed to treat the non-healing ulcers with platelet rich fibrin gel.

Materials and Methods: A total of 23 cases of non-healing ulcers were treated with autologous platelet rich fibrin gel once a week for 6 weeks. The pain reduction by VAS score, the mean area $\left(\mathrm{cm}^{2}\right)$ and the mean volume $\left(\mathrm{cm}^{3}\right)$ of the ulcer were analyzed at the end of 6 months.

Results: By the end of 4 months, almost 100\% improvisation in the ulcer area and its volume was seen in 17 (73.91\%) and 21 (91.30\%) of the ulcers respectively. The mean VAS score for ulcer showed a declining trend from $9.01 \pm 0.09$ to $2.19 \pm 1.32$ at the end of 6 months. The reduction of pain in the ulcer was due to the anti-inflammatory property of platelet rich fibrin matrix.

Conclusion: Platelet rich fibrin gel provides biological treatment for non- healing ulcers by decreasing the morbidity and improving the functional quality of life.
\end{abstract}

Keywords: Non-Healing Ulcer; Platelet; Fibrin 


\section{Introduction}

Ulcer is defined as wounds with a "full thickness depth" and a "slow healing tendency" and may result in complete loss of the epidermis and often portions of the dermis and even subcutaneous fat [1]. Chronic non-healing ulcer is defined as a persistent defect in the skin for the period of more than six weeks and does not exhibit any tendency to heal following three or more months. The symptomatologies of non-healing ulcers include increase in pain, friable granulation tissue, foul odor, and breakdown of wound rather progressing for healing. These results in social outcast and face more challenges in providing healthcare [2,3]. To reiterate, it not only represents as a considerable financial burden on the health care set-up of the country but also undermines the functional and productive outcome of the patients.

Tissue engineering has demonstrated regeneration potentiality without any permanent sequelae. Many researchers have proven that human skin has the ability to regenerate through available stem cell populations. Stem cell has the ability of self-renewal and ability to differentiate into function specific daughter cells. Regenerative medicine has a definite role in regulating these stem cell populations for promoting skin regeneration [4].

The contents of platelet-rich fibrin (PRF) include platelets, leukocytes, cytokines and adhesive proteins including fibrinogen, fibronectin, vitronectin, and thrombospondin-1 respectively $[5,6]$. It enhances wound healing through induction of locally accessible growth factors which favors recruitment and proliferation of mesenchymal stem cells (MSCs) and synthesis of extracellular matrix. Knighton., et al. demonstrated the aforementioned theory wherein utilization of platelet factors (autologous) resulted in complete repairing of the chronic non-healing ulcers by quickening the formation of granulation. He showed that natively acting biomolecules derived from autologous blood promoted healing of chronic ulcers present cutaneously [7]. In this article, we aimed to assess the functional outcome of autologous platelet rich fibrin therapy in nonhealing ulcers.

\section{Materials and Methods}

A total of 23 cases of non-healing ulcers for an observational study were recruited by convenient sampling technique. After seeking informed consent in written, all the enrolled cases in our study were directed to undertake local and systemic examination in view of determining the cause of their ulcer. The baseline labora- tory investigations and radiographic analysis of affected parts were done.

Preparation of platelet rich fibrin (PRF): A total of $20 \mathrm{cc}$ of venous blood was withdrawn into plain vacutainers for soft spin centrifugation at a rate of $2200 \mathrm{rpm}$ for the duration of 12 minutes. The resultant preparation in the vacutainer contained platelet rich fibrin gel in upper $2 / 3^{\text {rd }}$ part and RBCs in lower $1 / 3^{\text {rd }}$ part respectively. Platelet rich fibrin gel was separated from RBC layer.

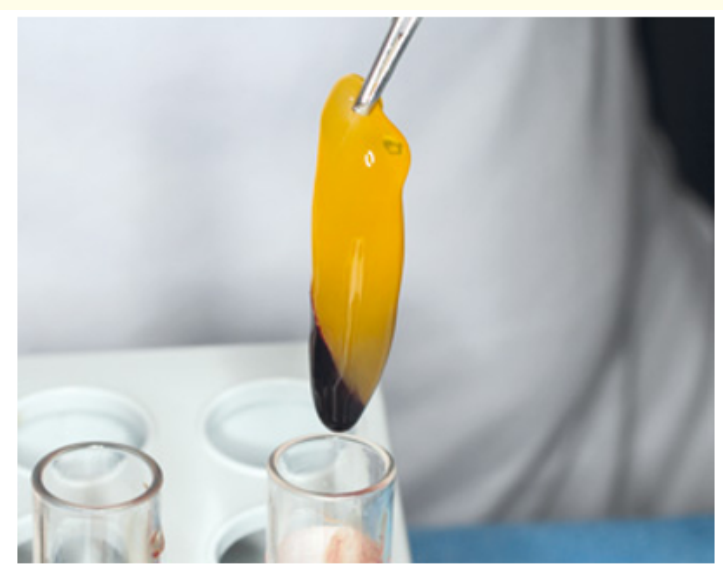

Figure 1: Platelet rich fibrin (PRF) gel.

After a thorough wound debridement, autologous platelet rich fibrin gel was applied across the ulcer and non-adhesive bandaging was done. After a duration of 1 week, ulcer was inspected. A total of 6 sittings of PRF application to ulcer at weekly intervals were done. The progress of natural course of non-healing ulcer and its management were monitored and charted in each case for statistical analysis.

\section{Results}

A total of 23 cases of non-healing ulcers of varying etiologies were treated using autologous platelet rich fibrin (PRF) at weekly intervals for maximum frequency of 6 sittings respectively. The mean age of the patients was $39.7 \pm 13.02$ years (As shown in table 1). Out of 23 ulcers, there were 7 (30.43\%) venous ulcers, $4(17.39 \%)$ traumatic ulcers, 7 (30.43\%) diabetic ulcers and 5 (21.73\%) trophic ulcers. The length of non-healing ulcer ranged 

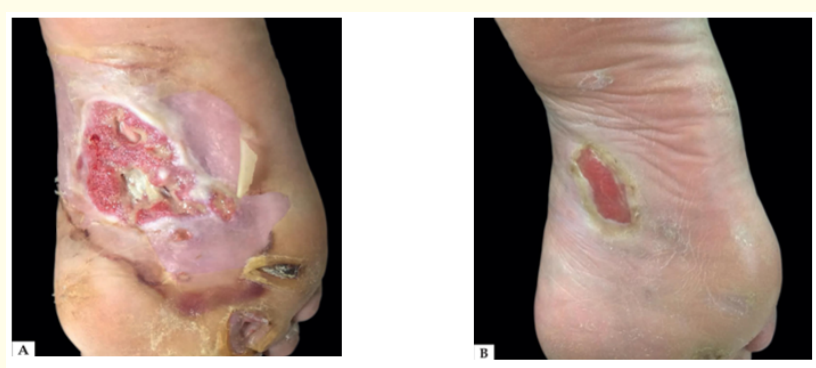

Figure 2: Non-healing ulcer over plantar aspect of foot

(A) Before treatment with PRF and (B) After treatment with 6 sittings of PRF dressings (at the end of 3 months).
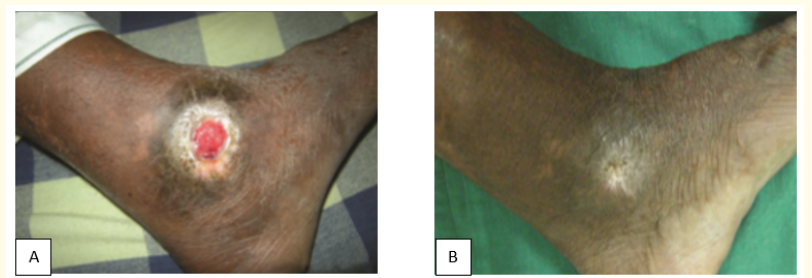

Figure 3: Non-healing ulcer over left medial malleolus (A) Before treatment with PRF and (B) After treatment with 6 sittings of PRF dressings (at the end of 3 months).

from 3 months to 14 months with a mean of $6.48 \pm 1.72$ months. The mean duration of ulcer healing was $4.81 \pm 3.45$ weeks.

The mean area $\left(\mathrm{cm}^{2}\right)$ and volume $\left(\mathrm{cm}^{3}\right)$ of the ulcer at baseline, at end of $3^{\text {rd }}$ and $6^{\text {th }}$ follow up were shown in table 2 . Almost $100 \%$ improvement in the area and volume of the ulcer was seen in 17 $(73.91 \%)$ and $21(91.30 \%)$ of the ulcers respectively at the end of the $3^{\text {rd }}$ month follow up. The mean VAS score for ulcer showed a declining trend from $9.01 \pm 0.09$ to $2.19 \pm 1.32$. The reduction of pain in the ulcer was due to the anti-inflammatory property of platelet rich fibrin matrix. We observed no significant complications in the entire duration of the study.

\section{Discussion}

Chronic non-healing ulcers due to various etiologies have become cumbersome and add morbidity for patients and society. These wounds pose a greater challenge for healthcare personnel to
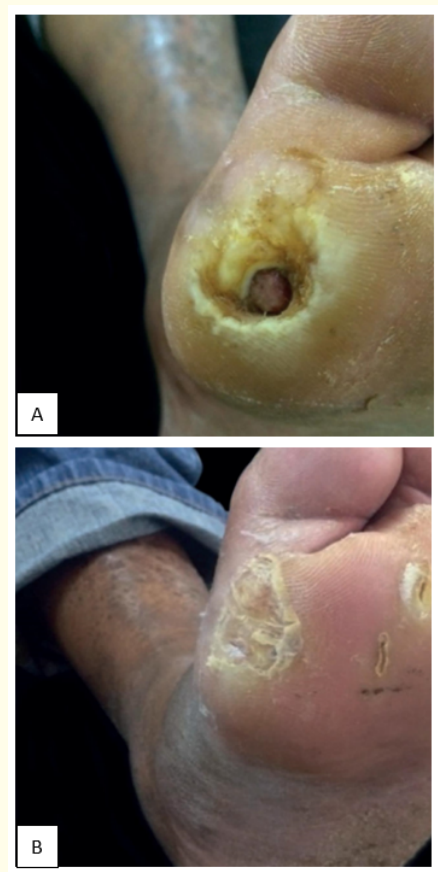

Figure 4: Non-healing ulcer over ball of 1st left metatarsal head (A) Before treatment with PRF and (B) After treatment with 6 sittings of PRF dressings (at the end of 3 months).

\begin{tabular}{|l|c|c|}
\hline $\begin{array}{c}\text { Age group (in } \\
\text { years) }\end{array}$ & Number of patients & Percentage (\%) \\
\hline $20-30$ & 3 & $13.04 \%$ \\
\hline $30-40$ & 2 & $8.69 \%$ \\
\hline $40-50$ & 8 & $34.78 \%$ \\
\hline $50-60$ & 7 & $30.43 \%$ \\
\hline $60-70$ & 3 & $13.04 \%$ \\
\hline
\end{tabular}

Table 1: Demographic details of patients.

treat. These usually present with exposed underlying tissues and further complicates the process of granulation tissue whilst simple dressing. Additionally, at the same time it presents with an equivocal challenge to maintain the viability of surrounding tissue. These non-healing ulcers need a multimodal approach for treating by ascertaining the underlying pathology and the systemic condition of the individual. Conventional approach of treatments were not able to provide growth factors to induce neovascularization at the ulcer 


\begin{tabular}{|l|c|c|c|}
\hline Variables & Baseline & $\mathbf{3}^{\text {rd }}$ follow up & $\mathbf{6}^{\text {th }}$ follow up \\
\hline Mean area $\left(\mathrm{cm}^{2}\right)$ & 11.32 & 4.74 & 1.02 \\
\hline CI & $\begin{array}{c}-1.942 \text { to } \\
4.037\end{array}$ & $\begin{array}{c}4.237 \text { to } \\
8.571\end{array}$ & 6.933 to 10.613 \\
\hline P value & 0.913 & $0.03^{* * *}$ & $<0.001^{* * *}$ \\
\hline $\begin{array}{l}\text { Mean volume } \\
\left(\mathrm{cm}^{3}\right)\end{array}$ & 7.43 & 2.19 & 0.21 \\
\hline CI & $\begin{array}{c}-1.426 \text { to } \\
3.965\end{array}$ & $\begin{array}{c}1.603 \text { to } \\
5.307\end{array}$ & 3.962 to 6.731 \\
\hline P value & 0.970 & $<0.001^{* * *}$ & $<0.001^{* * *}$ \\
\hline \multicolumn{4}{|l}{$* * *$ Statistically significant } \\
\end{tabular}

Table 2: $\mathrm{CI}$ and $\mathrm{P}$ value of mean area $\left(\mathrm{cm}^{2}\right)$ and mean volume $\left(\mathrm{cm}^{3}\right)$ of the ulcer.

site to initiate the healing process [8]. Thereby, we believe that such dilemmatic circumstances can be best dealt with newfangled approach based on using platelet concentrates.

Regenerative medicine dogma embraces therapeutic potentiality in induction of stem cells through locally available biomolecules to promote skin regeneration and rejuvenation [9]. Dr. Joseph Choukroun, a research pioneer developed second-generation platelet concentrate wherein anticoagulation factors were not utilized. It involves subjection of peripheral blood to one-step based centrifugation either at $3000 \mathrm{rpm}$ for 10 minutes or $2700 \mathrm{rpm}$ for 12 minutes without application of anticoagulants and blood activating substances. Briefly, venous blood collected in a glass tube without anticoagulants is centrifuged at low speed and the resultant yield is a three layered solution. The middle fraction of this product has a fibrin clot which is the biological product of interest and can be readily applied at the wound site in its membranous formulation (fibrin matrix). The notable constituents of PRF are white blood cells concentrates, platelets, and fibrin. It has been shown that the initially developed Platelet rich fibrin (also termed L-PRF) composed of $97 \%$ platelets and more than 50\% leukocytes in a high-density fibrin network when compared to whole blood [10-12].

Gel formulation of platelet rich fibrin is not conducive and hence for overcoming this limitation several modifications have been done and now newer formulations have been introduced $[13,14]$.
PRF contains enormous platelets with fibrin. Once alpha granules in the platelets start degranulation, it releases various growth factors (transforming growth factor $\beta$, platelet derived growth factor, epidermal growth factor, nerve growth factor) along with vitronectin, fibronectin and sphingosine 1-phosphate which helps in enhancement of wound healing and microenvironment homeostasis [15]. This unique organization in the form of the 3-dimentional fibrin matrix provides a binding site for platelets as well as growth factors. This flexible mesh serves as scaffold to promote cellular migration in micro-environment and perquisite in repairing and regenerating tissue. Overall, in PRF preparation, leukocyte and fibrin acts as mutual stimulatory actors by imitating the physiological process of wound healing and boosting angiogenic, osteogenic and antimicrobial activities [16].

The biological characterization of platelet rich plasma and platelet rich fibrin has been tabulated (Table 3). Suryanarayan S., et al. showed the mean duration of ulcer healing with PRP was 5.6 weeks [17]. Kim SA., et al. showed 90 - 100\% epithelization after 15.18 days of treatment with PRP for non-healing ulcers [18]. Frykberg., et al. showed that 63 of 65 ulcers responded with a reduction in area, volume and undermining of the ulcers in a mean duration of 2.8 weeks with PRP treatments [19]. In our study, the mean duration of healing of non-healing ulcers with PRF was $4.81 \pm 3.45$ weeks. The mean area $\left(\mathrm{cm}^{2}\right)$ and volume $\left(\mathrm{cm}^{3}\right)$ of the ulcer treated with PRF was statistically significant at the end of $3^{\text {rd }}$ month follow up. Almost $100 \%$ improvisation in the ulcer area and its volume was seen in 17 (73.91\%) and 21 (91.30\%) of the ulcers respectively at the end of the $3^{\text {rd }}$ month follow up.

We observed a commendable outcome in all the ulcers without any adverse side effects. The treatment of non-healing ulcer with this platelet concentrate is a breakthrough in terms of challenges faced during the treatment and morbidity in the quality of life of the patients. This method is comparatively fast, cost-effective and applicable without any requirement of hospitalization with benefit of reduction in hours lost for work. However, the major limitation of our study is no inclusion of control group for comparing PRF applications and therefore considering it as a 'holy grail' for treating non-healing complex wounds solely seems indefensible. The other notable limitations include smaller sampling size, and single-centered study respectively. 


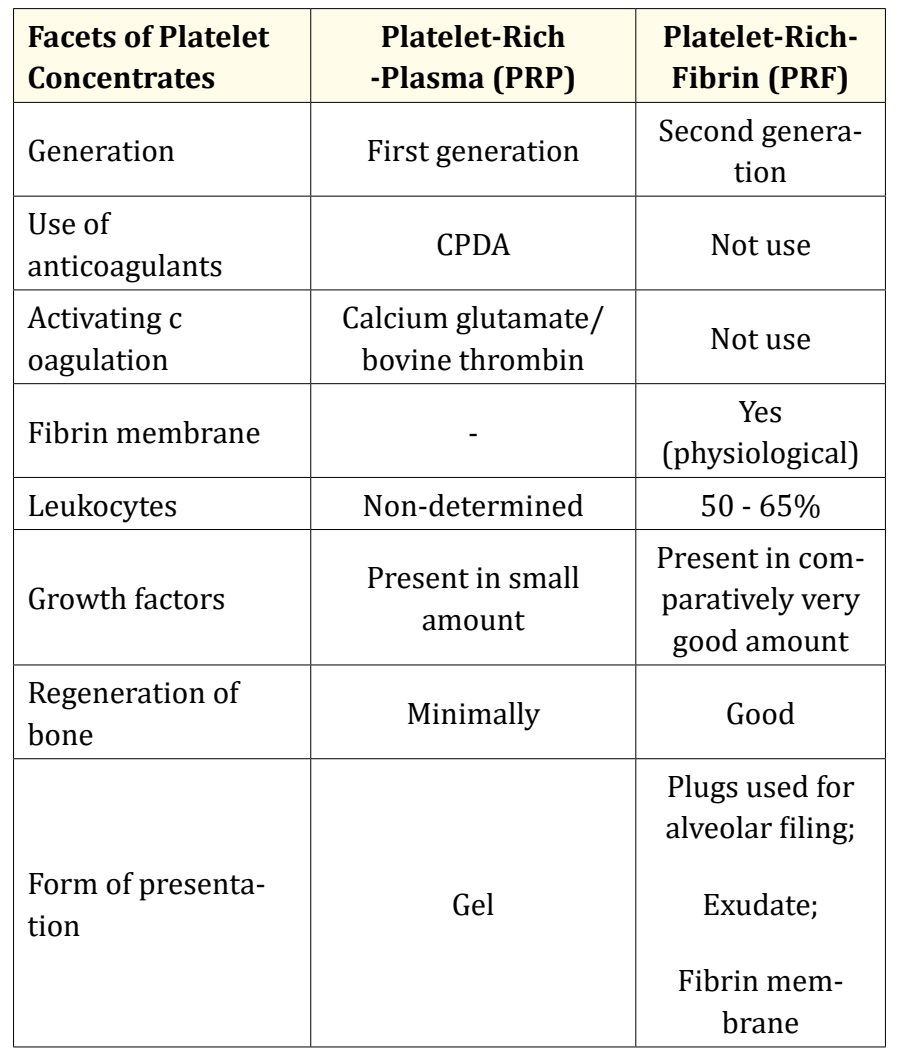

Table 3: Schematic tabulation of biological characterization of PRP vs PRF [16].

\section{Conclusion}

Chronic non-healing ulcers pose a major threat to public in terms of social stigma and social outcast in developing countries. Regenerative medicine holds a promise in treating the difficult non-healing ulcers with induction of locally available biomolecules. In our study, we used PRF to treat non-healing ulcers. There is a paucity of available literature to prove the superiority of PRF over PRP. Further, randomized controlled trials are necessary to demonstrate PRF's efficacy, superiority over PRP and to be considered as armamentarium for treating non-healing ulcers.

\section{Bibliography}

1. Shubhangi Vinayak Agale. "Chronic Leg Ulcers: Epidemiology, Aetiopathogenesis, and Management (2013): 9.

2. B Kahle., et al. "Evidence-based treatment of chronic leg ulcers". Deutsches Ärzteblatt International 108.14 (2011): 231237.
3. WB Van Gent., et al. "Management of venous ulcer disease". The British Medical Journal 341.7782 (2010): 1092-1096.

4. Victor W Wong., et al. "Stem Cell Niches for Skin Regeneration (2012): 8.

5. Choukroun J., et al. "Platelet-rich fibrin (PRF): a second-generation platelet concentrate. Part V: histologic evaluations of PRF effects on bone allograft maturation in sinus lift". Oral Surgery, Oral Medicine, Oral Pathology, Oral Radiology, and Endodontology 101.3 (2006): 299-303.

6. Choukroun J., et al. "Platelet-rich fibrin (PRF): a second-generation platelet concentrate. Part IV: clinical effects on tissue healing". Oral Surgery, Oral Medicine, Oral Pathology, Oral Radiology, and Endodontology 101.3 (2006): e56-60.

7. Knighton DR., et al. "Classification and treatment of chronic nonhealing wounds. Successful treatment with autologous plateletderived wound healing factors (PDWHF)". Annals of Surgery 204 (1986): 322330.

8. GA Rahman., et al. "Epidemiology, etiology, and treatment of chronic leg ulcer: experience with sixty patients". Annals of African Medicine 9.1 (2010): 1-4.

9. VW Wong., et al. "Stem cell niches for skin regeneration". International Journal of Biomaterial (2012): 8.

10. Dhurat R and Sukesh M. "Principles and Methods of Preparation of Platelet-Rich Plasma: A Review and Author's Perspective". Journal of Cutaneous and Aesthetic Surgery 7 (2014): 189-197.

11. Chandran P and Sivadas A. "Platelet-rich fibrin: Its role in periodontal regeneration". Journal of Dental Research 5 (2014): 117-122.

12. Ghanatti S., et al. "Advanced platelet rich fibrin a new concept of cell based tissue engineering by means of inflammatory cells". The Journal of Oral Implantology 6 (2014): 679-689.

13. Mourao CF., et al. "Obtention of injectable platelets rich-fibrin (i-PRF) and its polymerization with bone graft: technical note". Revista do Colégio Brasileiro de Cirurgiões 42 (2015): 421-423.

14. Kobayashi E., et al. "Comparative release of growth factors from PRP, PRF, and advanced-PRF+". Clinical Oral Investigations 2 (2016): 23-30. 
15. Tunalı M., et al. "In vivo evaluation of titanium-prepared platelet-rich fibrin (T-PRF): a new platelet concentrate". British Journal of Oral and Maxillofacial Surgery 51 (2013): 438-443.

16. Caruana A., et al. "From Platelet-Rich Plasma to Advanced Platelet-Rich Fibrin: Biological Achievements and Clinical Advances in Modern Surgery". European Journal of Dentistry 13.2 (2019): 280-286.

17. Suryanarayan S., et al. "Efficacy of autologous platelet-rich plasma in the treatment of chronic nonhealing leg ulcers". Plastic and Aesthetic Research 1 (2014): 65-69.

18. Kim SA., et al. "Application of platelet-rich plasma accelerates the wound healing process in acute and chronic ulcers through rapid migration and upregulation of cyclin A and CDK4 in HaCaT cells". Molecular Medicine Reports 7 (2013): 476-480.

19. Frykberg RG., et al. "Chronic wounds treated with a physiologically relevant concentration of plateletrich plasma gel: a prospective case series". Ostomy Wound Manage 56 (2010): 3644.

Volume 4 Issue 9 September2021

(C) All rights are reserved by Madhan Jeyaraman., et al. 